\title{
OSTEOPOROSIS AND EXERCISE
}

\author{
Roger L Wolman
}
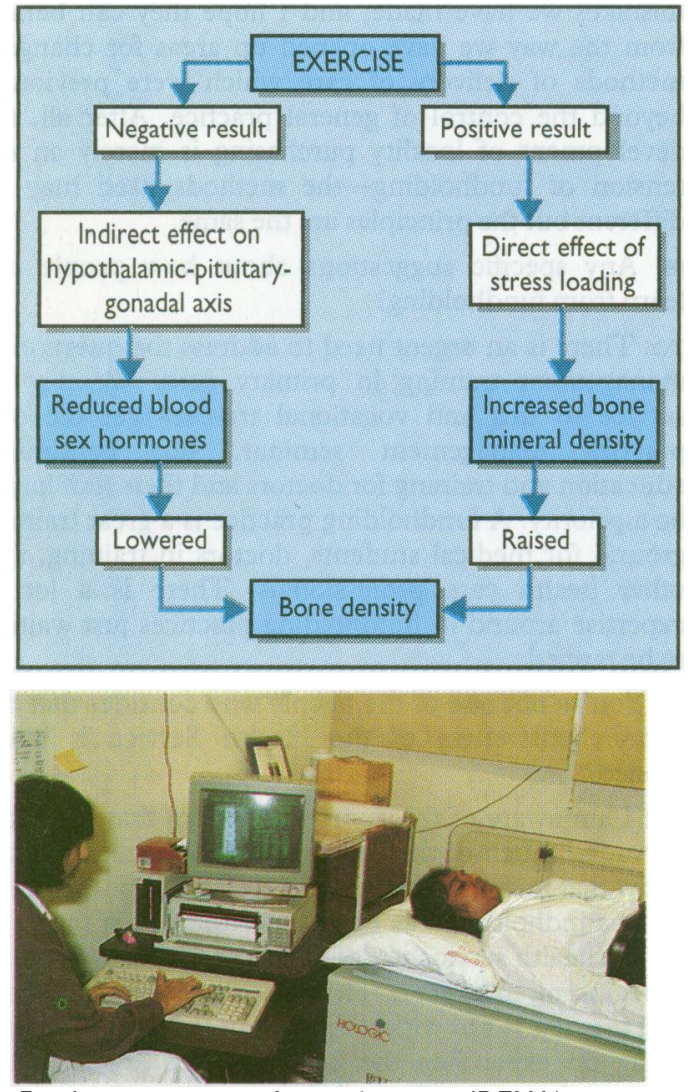

Dual energy $x$ ray absorptiometry (DEXA) measurement of bone density.
Exercise affects the skeleton in several ways. The direct effect of stress loading can be to increase bone mineral density, and this is now being considered as a strategy to prevent osteoporosis. Intensive aerobic exercise, however, can adversely affect bone density indirectly by its effect on the hypothalamic-pituitary-gonadal axis, which leads to a fall in blood oestrogen concentrations. This article discusses these effects.

For 100 years it has been known that bone tissue adapts to the stress loads to which it is exposed. During the past 20 years bone densitometry has made it possible to assess the effects of physical activity on the skeleton. Bone densitometry measures the calcium concentration within bone. Several methods are used to measure bone density: the most popular is dual energy $x$ ray absorptiometry (DEXA) because it gives a highly reproducible result and exposes patients to only small amounts of radiation. It uses a pencil-thin beam of $x$ rays that scan over a skeletal site to a detector on the opposite side of the patient. From this, an estimate is obtained of the calcium concentration of the scanned bone.

\section{Factors that increase bone density}

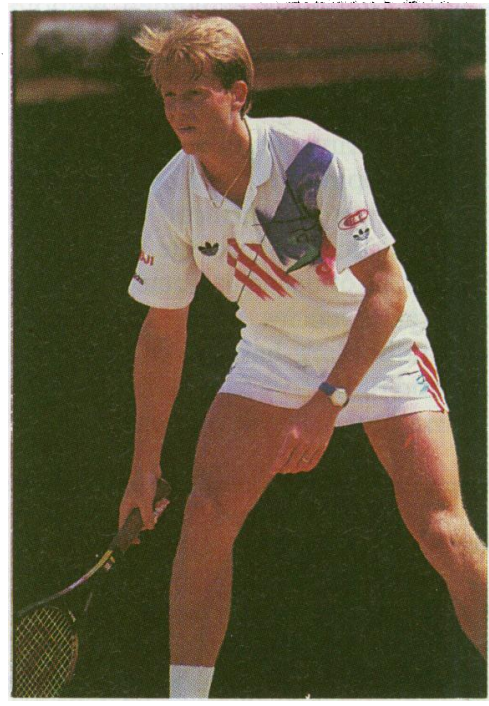

Stefan Edberg's playing arm is considerably thicker than his nonplaying arm.

\section{Gravity (weight bearing)}

Low levels of physical activity lead to a fall in bone density. Complete bed rest gives a negative calcium balance within a few days and a detectable reduction in bone density within a few weeks. This detrimental effect emphasises the importance of early mobilisation after acute illness or operation. The lack of gravity stimulation is thought to be responsible for the losses seen in the gravity free environment of astronauts. Though regular exercise is associated with an increase in bone density, this benefit is less pronounced if the exercise does not incorporate gravity stimulation. Thus swimming, being a weight supported activity, produces only a limited effect on the skeleton compared with some weight bearing activities.

\section{Anatomical site of maximum stress}

The skeletal response to exercise is greatest at the site of maximum stress. This effect is seen well in professional tennis players, whose playing arm can be up to $30 \%$ more dense than the non-playing arm. Large increases in bone density are seen at certain skeletal sites in athletes from other sports. For example, runners have increased bone density in the os calcaneous, femoral shaft, and spine compared with sedentary controls. Rowers, who perform intensive upper body exercise, display even more appreciable increases in spinal bone density. 


\begin{tabular}{|ll|}
\hline $\begin{array}{l}\text { Physical } \\
\text { activity }\end{array}$ & $\begin{array}{l}\text { Site of bone } \\
\text { density increases }\end{array}$ \\
\hline $\begin{array}{l}\text { Running } \\
\text { (jogging) }\end{array}$ & $\begin{array}{l}\text { Os calcaneous, tibia, } \\
\text { shaft of femur, spine } \\
\text { Dominant arm, spine }\end{array}$ \\
$\begin{array}{l}\text { Tennis } \\
\text { Rowing }\end{array}$ & $\begin{array}{l}\text { Spine } \\
\text { Volleyball }\end{array}$ \\
Basketball & Spine, os calcaneous \\
& Spine, os calcaneous \\
\hline
\end{tabular}

\section{Adverse effects of intensive training}
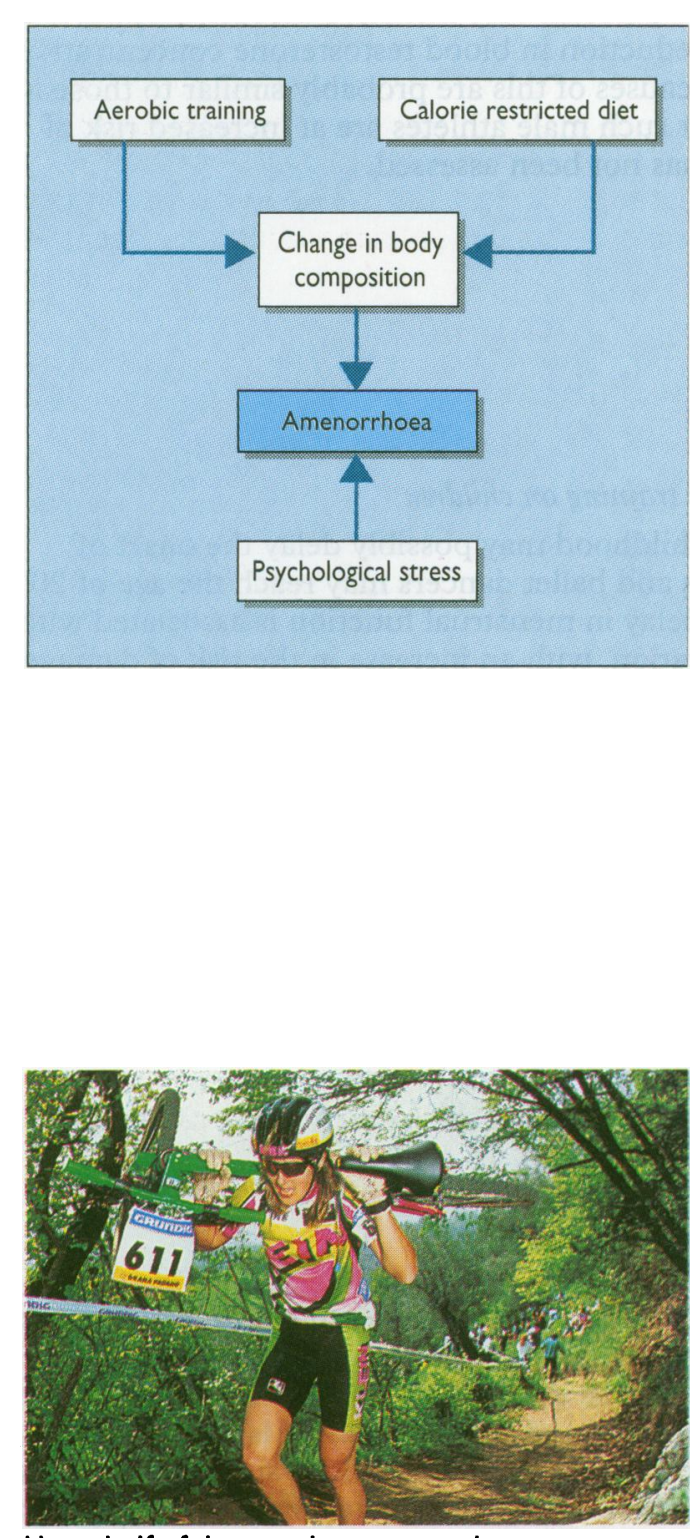

Up to half of the top class women have amenorrhoea and thus risk osteoporosis. falls.

\section{Repetitive movements}

Any exercise activity that produces repetitive stress loading to a part of the skeleton will tend to increase bone density at that site. The running action produces repetitive stress loading of the legs (800-2000 strides per mile $(1.6 \mathrm{~km})$ run - that is, $400-1000$ repetitive loads on each leg), and this explains the impressive gains in leg bone density seen in long distance runners. Aerobic training, which usually entails continuous repetitive movements of two or more limbs, is associated with increases in bone density. Strength training, by stress loading a part of the body to build up muscle strength, also increases bone density.

\section{Benefits of repetitive weight-bearing exercise}

Intervention studies have shown that the prescription of these sorts of exercise programmes can lead to appreciable increases in bone density after several months of training. For older people exercise may reduce the risk of osteoporosis, even to people in their 80s. Exercise is not as effective as oestrogen replacement in postmenopausal women, but in older people exercise may also reduce the risk of osteoporotic fractures by improving muscle tone and balance, so reducing the risk of

\section{"Athletic amenorrhoea" in women}

Aerobic exercise can affect the hypothalamic-pituitary-gonadal axis, thus leading to a reduction in oestrogen and progestogen release from the ovaries. The menstrual cycle may then become irregular or even cease. This "athletic amenorrhoea" was first described in the late '70s. Before then it was unusual for women to train sufficiently hard to develop amenorrhoea. With the increase in popularity of women's marathons, triathlons, and other endurance events, this disorder is becoming increasingly common.

\section{Causes of hypothalamic-induced amenorrhoea}

The cause of hypothalamic-induced amenorrhoea is uncertain. It is related to training intensity and to calorie restriction in the diet. A combination of these two factors leads to changes in body composition, in particular a reduction in body fat, and this seems to be a major influence in the development of amenorrhoea. Psychological stress may be a contributory factor, especially in competitive athletes. The incidence varies and is a reflection of the training requirements for each sport. Up to a half of the top class ballerinas, cyclists, runners, and rowers are amenorrhoeic.

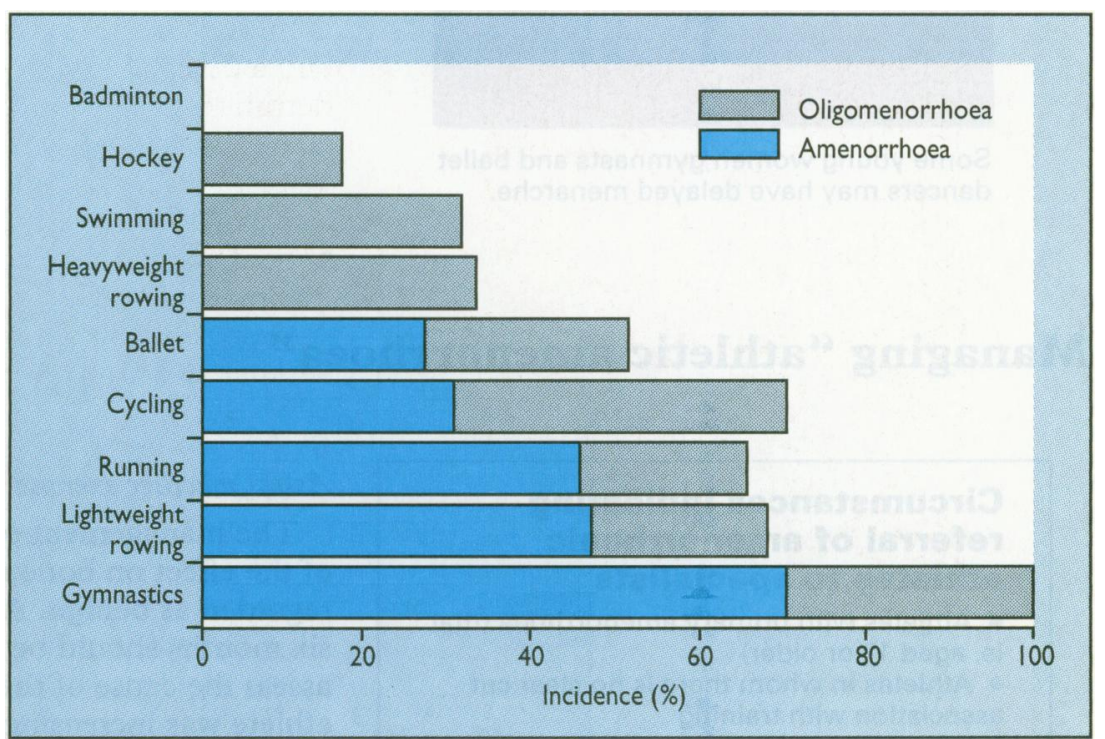

Proportions of women in various sports who have reduced or absent menstruation. 


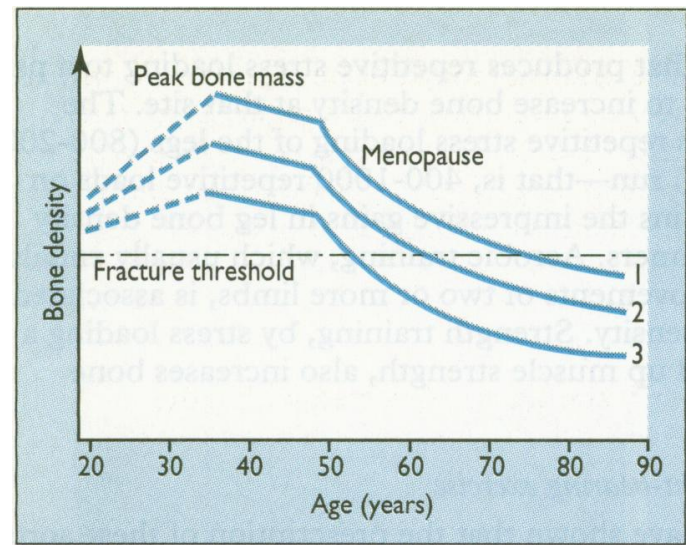

Bone density changes with age in women. Line $2=a n$ average woman. Prolonged amenorrhoea in the 20 s is likely to increase the risk of osteoporosis in later life (line 3). Conversely, intensive exercise in the 20 s may reduce this risk (line 1).

\section{Skeletal associations with low oestrogen concentration}

- Reduced bone mineral density

- Stress fractures

- Delayed epiphyseal closure in adolescent athletes

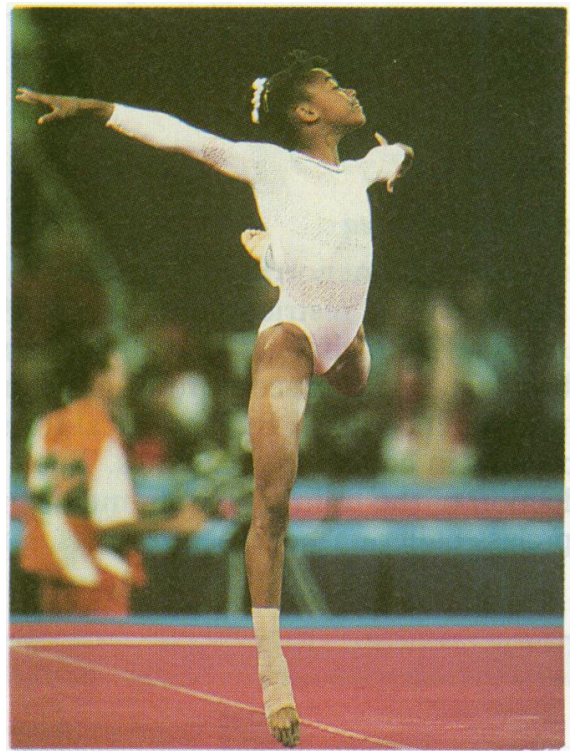

Some young women gymnasts and ballet dancers may have delayed menarche.

\section{Effect of low blood oestrogen concentration on bone}

Low oestrogen concentration has several effects on the skeleton, in particular a reduction in bone density despite high levels of exercise. The most important reduction occurs at sites where trabecular bone predominates (the spine and, to a lesser extent, the proximal femur and distal radius). With short episodes of amenorrhoea (up to six months) the fall in bone density is reversible, but if amenorrhoea is prolonged (two to three years) the loss may become irreversible. The risk of osteoporosis in later life is then likely to increase. In some athletes with prolonged amenorrhoea loss of bone density is pronounced and puts them at immediate risk of fracture. There have been some reports of insufficiency fractures occurring in athletes in their $20 \mathrm{~s}$.

Amenorrhoeic athletes have a higher incidence of stress fractures than their eumenorrhoeic counterparts. Although this may be due to low oestrogen, intensive training increases the risk of both stress fractures and amenorrhoea.

\section{Effects of intensive aerobic training on men}

There is some evidence to show that hypothalamic-pituitary-gonadal axis function in men who perform very intensive aerobic training is also disturbed, leading to a reduction in blood testosterone concentrations and sperm counts. The causes of this are probably similar to those in female athletes. Whether such male athletes are at increased risk of osteoporosis, however, has not been assessed.

\section{Effects of intensive aerobic training on children}

Intensive training in childhood may possibly delay the onset of puberty. Some gymnasts and ballet dancers may reach the age of 20 before menarche. This delay in menstrual function is associated with effects on skeletal maturation, with an increase in the risk of damage to the epiphysis.

\section{Managing "athletic amenorrhoea"}

\section{Circumstances indicating referral of amenorrhoeic athletes to specialists}

- Athletes with primary amenorrhoea (that is, aged 17 or older)

- Athletes in whom there is no clear cut association with training

- When amenorrhoea persists for more than six months

\section{Assess whether exercise is the cause}

The management of amenorrhoeic athletes can be difficult. In view of the effect on bone mineral density, this condition should not be regarded as benign. Any female athlete who has been amenorrhoeic for six months should be assessed medically. Initially it is important to assess the cause of the amenorrhoea. If her periods stopped when the athlete was increasing her training and started again when her training decreased (for example, during an injury) then the exercise was probably the cause. 
The relation with exercise intensity may not be clear cut, in which case a formal gynaecological opinion should be sought. Bone density measurement is important to calculate the degree of bone mineral loss. A nutritional assessment is also helpful as it will show whether the athlete's diet is deficient in calories or calcium, or both.

\section{Managing amenorrhoeic athletes}

- Gynaecological assessment

- Nutritional assessment

- Reduction in training intensity

- Increase in calorie intake

- Consider oestrogen replacement (oral contraceptive or hormone replacement therapy)

\section{Treatment options}

Dietary calcium deficiency should be corrected with either supplements or foods rich in calcium. Increasing the calorie intake may restore menstruation without an appreciable impairment in performance. The most effective way to treat this condition, however, is to get the athlete to reduce training intensity. Many will be unwilling to do this, in which case oestrogen replacement, in the form of either the oral contraceptive or hormone replacement therapy, should be considered. Some athletes are unable to tolerate hormone treatment, however, because of problems with fluid retention and the premenstrual syndrome. By this stage therapeutic options are limited, but at the very least athletes need to be warned about the potential long term risks associated with amenorrhoea.

\section{Conclusion}

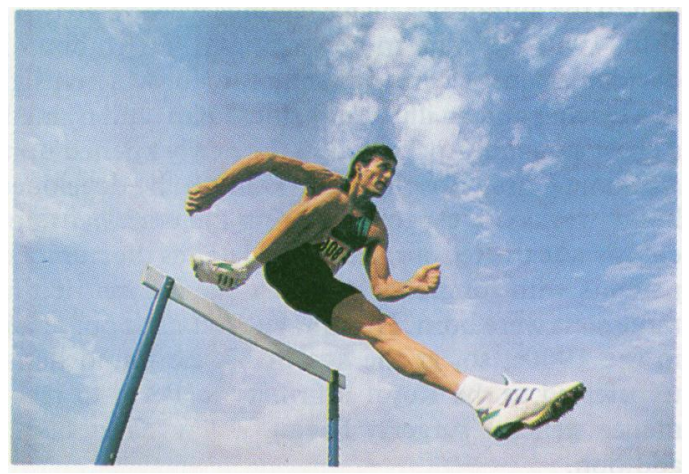

The photograph of Stefan Edberg is reproduced with permission of Colorsport, that of
Exercise provides an additional method for preventing and treating osteoporosis. Athletes tend to have higher bone densities than the general population and can provide a model for assessing the effects of different exercise regimens on bone mineral density. Intensive aerobic training can, paradoxically, lead to a fall in bone density caused by low oestrogen. The exercise and oestrogen levels of a woman in her $20 \mathrm{~s}$ and 30 s may be very important in determining her eventual risk of developing osteoporosis. the cyclist with permission of Professional Sport, that of the gymnast with permission of Allsport, and that of the hurdler with permission of Colorsport.

Roger Wolman is consultant in sports medicine and rheumatology at the Royal National Orthopaedic Hospital Trust, Stanmore, Middlesex HA7 4LP.

The ABC of Sports Medicine has been edited by Greg McLatchie, visiting professor of sports medicine and surgical sciences at the University of Sunderland, consultant surgeon at Hartlepool General Hospital, and director of the National Sports Medicine Institute, London. This paper has been edited in conjunction with Mark Harries, consultant physician at Northwick Park Hospital, Harrow, and director of clinical services at the British Olympic Medical Centre, Harrow.

\section{A MEMORABLE PATIENT The privilege of teaching}

She was a middle aged Ugandan woman with a much older face. She was the patient on whom Helen Diziciru, an ophthalmic nurse, performed her first complete cataract operation in April this year. I was supervising. We can all remember the thrill the first time we finished an operation or a procedure by ourselves. My first appendicectomy was performed after a completely sleepless night on call, but I remember every detail. Helen's cataract patient lay perfectly motionless for one and a half hours. On the next table the intracapsular cataract extractions were taking less than 15 minutes each. There was a moment of tension as Helen tumbled the cataract out of the eye so neatly you wondered how it had stayed in there for 50 years.

With this method of intracapsular cataract extraction known as "tumbling" there is a moderate risk of some of the vitreous humour also being extracted inadvertently, especially when you are beginning to learn the surgery. This vitreous loss carries with it a subsequent risk of retinal detachment. Helen's patient's vitreous remained in its place thank God.
Helen relaxed, remembered to breathe again, and started painstakingly taming the fine silk suture material we used to close the wound. This microscopic suture is thinner than a hair and without a microscope it seemed to have a rebellious life of its own. Helen was using a loope, a magnifying device attached to spectacles, but these have a narrow field of vision and the suture was apparently jumping in and out of her view like a Jack in the box. Many prolonged minutes later the wound was closed.

The next day our patient received the thick cataract glasses that patients used to be given in Britain not so long ago. Her elderly face creased into lines and crevices as she smiled and laughed and gave thanks.

Having been only a senior house officer before I went to Uganda I had never before had to take anyone through an operation. This memorable patient taught me something of the stress of a consultant supervising in the theatre, and also something of the privilege it is to teach-when it goes well. This patient's joy the next day at being able to see also taught me again that even short term service in Africa, if the emphasis is on teaching, can be worth while.-AIDEEN LANDERS is a locum registrar in ophthalmology in London. 